\title{
Use of Survival Analysis to Determine the Postincubation Time-to-Death of Papaya Due to Yellow Crinkle Disease in Australia
}

\author{
Paul D. Esker, Department of Plant Pathology, Iowa State University, Ames 50011; Karen S. Gibb, School of Bio- \\ logical, Environmental and Chemical Sciences, Charles Darwin University, Darwin 0909, Australia; Anna Padovan, \\ CSIRO Tropical Ecosystems Research Centre, PMB44 Winnellie 0822, Australia; Philip M. Dixon, Department of \\ Statistics, Iowa State University, Ames 50011; and Forrest W. Nutter, Jr., Department of Plant Pathology, Iowa \\ State University, Ames 50011
}

\begin{abstract}
Esker, P. D., Gibb, K. S., Padovan, A., Dixon, P. M., and Nutter, F. W., Jr. 2006. Use of survival analysis to determine the postincubation time-to-death of papaya due to yellow crinkle disease in Australia. Plant Dis. 90:102-107.

The current management recommendation for papaya (Carica papaya) plants exhibiting symptoms of yellow crinkle disease in Australia is the practice of ratooning infected plants. Ratooning involves removing the main stem of diseased papaya plants and allowing a lateral stem (supposedly pathogen-free) to develop and replace the diseased stem. Using nonparametric and parametric methods of survival analysis, we tested different hypotheses regarding plant factors that may influence the postincubation period survival time of phytoplasma-infected papaya. The factors included plant age, the season (wet versus dry) when papaya plants first became symptomatic, and the two predominant phytoplasma strains causing papaya yellow crinkle: tomato big bud (TBB) or sweet potato little leaf strain V4 (SPLL-V4). Median survival time was estimated to be from 4 to 5 months. Therefore, we estimated that the infectious period (incubation period plus the period from postincubation to time-to-death period) of infected papaya ranges from 6 to 9 months. Using parametric accelerated failure modeling and nonparametric Cox proportional hazard modeling, no significant improvement from a null model (no covariates) was found when analyzing plant age, the season a plant was observed to be symptomatic, or phytoplasma strain. However, the season in which a papaya plant became symptomatic differed between the two phytoplasma strains, indicating that the TBB and SPLL-V4 strains may have different modes of insect acquisition and transmission. Because of the long infectious period and the rate of plantto-plant spread, we question the use of ratooning as the primary management tactic for managing papaya yellow crinkle.
\end{abstract}

Additional keywords: Kaplan-Meier survival functions

Papaya (Carica papaya L.) diseases caused by phytoplasmas have been responsible for severe epidemics in papaya plantations in the Northern Territory, Australia $(3,10,16)$. There are three major diseases of papaya caused by phytoplasmas, and all three occur in Australia: dieback, papaya yellow crinkle, and papaya mosaic (3). Padovan and Gibb (16) observed that there were two predominant phytoplasma strains causing papaya yellow crinkle disease in the Northern Territory. Based on the use of restriction fragment length polymorphism (RFLP) analysis, papaya yellow crinkle was found to be associated with either the tomato big bud (TBB) or the sweet potato little leaf strain V4 (SPLL-V4). These two phytoplasma strains were previously considered to be indistinguishable from pa-

Corresponding authors:

P. D. Esker and F. W. Nutter, Jr.

E-mail: pde@iastate.edu and fwn@iastate.edu

Accepted for publication 2 September 2005.

DOI: 10.1094/PD-90-0102

(C) 2006 The American Phytopathological Society paya yellow crinkle and papaya mosaic, respectively, based on RFLP analysis $(4,9)$. However, based on 16S rRNA sequences, Schneider et al. (19) differentiated the two strains and placed both TBB and SPLL-V4 into the faba bean phyllody strain cluster.

The primary management tactic for phytoplasma-infected papaya involves the practice of ratooning of infected plants (11). In this management practice, symptomatic plants are cut close to ground level, and a lateral bud, assumed to be free of phytoplasma, is allowed to sprout and grow. The principal reason for ratooning is the assumption that the lower lateral bud(s) is pathogen-free (11). However, Guthrie et al. (11) reported that if the phytoplasma strains causing papaya yellow crinkle and papaya mosaic are detected within the roots and main stem of the plant, the assumption that lateral buds will be phytoplasma-free is false, and therefore, ratooning will not be an effective management practice.

Padovan and Gibb (16) reported that the mean survival time for phytoplasmainfected papaya (after symptom expression) was $4.3 \pm 2.2$ months (median sur- vival $=3.5$ months) for the TBB strain and $3.9 \pm 2.2$ months (median survival $=3.3$ months) for the SPLL-V4 strain (15). However, these values were calculated only for plants that were symptomatic (infected) and then died during the study period, whereas a number of infected papaya plants had not died at the time when the final disease incidence assessment was performed (month 36). Such data are referred to as "censored data" because they are observations that do not fail (i.e., die) within the time frame of the study and therefore were not included in statistical analyses. Survival analysis is a method that enables the analysis of all the survival data (i.e., the population of plants that were infected and died, plus the population of plants that were infected but did not die by the end of the study). Survival analysis statistical methods are commonly used in ecological and medical experiments to analyze the time-to-death (or time-toevent) data $(2,5,8,12,14)$. These methods offer several advantages over ANOVA methods in that they can handle repeated measurements over time on the same sampling units, including censored observations, and account for failure times (i.e., death) that are not normally distributed (8). In contrast to the medical and ecological fields, survival analysis has rarely been applied in plant pathology $(13,18,21)$.

Scherm and colleagues $(17,18)$ stated that plant pathology research data are often collected in the form of time-to-event data (e.g., time to germination, infection, symptoms, and/or death). The major advantage of using survival analysis is the inclusion of censored data in the analysis. In the papaya-phytoplasma pathosystem, for example, this may permit the inclusion of infected plants that do not die within the time period that data were collected, thus providing a more realistic estimate of postsymptomatic survival time. Including such plants in the analysis is important during an epidemic because these plants will continue to be infectious (i.e., serve as a source of inoculum for vectors to acquire and then transmit the pathogen to noninfected plants).

Padovan and Gibb (16) have suggested that time-to-death of phytoplasma-infected papaya may be influenced by plant age and/or the season in which a papaya plant 
is infected (i.e., wet season versus dry season), but did not test this hypothesis. Because plant age and season may affect proposed management tactics, including if and when ratooning is appropriate as a management practice, the capability of survival analysis to statistically address these questions further justifies the use of survival analysis. Therefore, the objectives of this study were to investigate the use of survival analysis methods to estimate postincubation period time-to-death of phytoplasma-infected papaya causing yellow crinkle disease and to assess the effect of three factors on time-to-death of phytoplasma-infected papaya plants: (i) whether time-to-death is similar for infection by either TBB and SPLL-V4 phytoplasma strains, (ii) whether plant age at time symptoms are first observed influences time-to-death, and (iii) whether time of season (wet or dry) during which a papaya plant first became symptomatic influences time-to-death.

\section{MATERIALS AND METHODS}

Study area and data collection. The field design and sampling methods used to study the effect of phytoplasmas on papaya plants have been previously described by Padovan and Gibb (16) and will be summarized here. The site used for the field study was an on-farm experiment located $350 \mathrm{~km}$ south of Darwin in the Northern Territory, Australia. Papaya seedlings were obtained commercially by the grower, and the plantation was established in January 1996. The plantation measured 65 rows by 55 plants per row with rows spaced $2.5 \mathrm{~m}$ apart and plants within rows spaced $2 \mathrm{~m}$ apart. Based on these dimensions, approximately 3,500 papaya plants were monitored in this study. No testing of papaya seedlings for phytoplasmas was performed at the beginning of the study. Beginning in May 1996, all plants were visually inspected each month for disease symptoms typical of those associated with phytoplasmas (dieback, yellow crinkle, and mosaic). Leaf samples collected from each symptomatic plant were tested for the presence of the phytoplasma strains causing papaya yellow crinkle or papaya dieback using a polymerase chain reaction (PCR) method (16). Identification of specific strains of phytoplasmas was accomplished using RFLP (16). Papaya plants were assessed monthly from May 1996 $($ month $=5)$ to April 1999 (month $=40)$. The month, row number, and plant number within the row when each infected papaya plant was first observed to be symptomatic (and also tested positive by PCR) was recorded. The infected papaya plants were monitored monthly until plant death occurred. For the survival analysis, we defined time-to-death as the month in which a plant was symptomatic and also tested positive for a phytoplasma strain, to the time (month) that plant death occurred.
Two events during this study occurred that constituted the censoring events. The first occurred at the end of September 1998 when 21 rows in the plantation were removed. Plants that had not died within those 21 rows had censored survival times determined to September 1998 (month = 33). The second censoring event occurred in April 1999 (month = 40), when the study was concluded. Plants that were symptomatic and had tested positive by PCR, but were not dead, were considered censored since the time-to-death was unknown.

Kaplan-Meier estimate of survival function. To test the hypotheses that (i) survival time was not affected by phytoplasma strain (TBB and SPLL-V4) and (ii) survival time was not influenced by whether plants became symptomatic (and tested positive with PCR) during the wet season or dry season, we compared the Kaplan-Meier estimates of survival probabilities using the log-rank test $(2,12)$. The Kaplan-Meier estimate of the survival function is a nonparametric procedure. The survival function is the number of individuals with survival time $\geq t$ (time) divided by the number of individuals in the study $(2,12)$. Survival times were calculated as the month in which a plant died minus the month in which a plant was first found to be symptomatic and tested positive by PCR. The Kaplan-Meier estimate of the survival function is a product-limit estimate:

$$
\tilde{S}(t)=\prod_{j=1}^{k}\left(\frac{n_{j}-d_{j}}{n_{j}}\right)
$$

where $n_{j}=$ number of individuals alive just before time $t_{(j)}$, and $d_{j}=$ number of deaths at $t_{(j)}$ for $t_{(k)} \leq t \leq t_{(k+1)}$ and $k=1,2, \ldots, r$. Because survival data tend to be right and positively skewed, the median survival time was estimated instead of mean survival time (2). The median survival time is defined as the time in which $50 \%$ of individuals in the study are expected to survive (2). Survival time data were analyzed using the LIFETEST procedure in SAS v. 8.0 (SAS Institute, Cary, NC) with phytoplasma strain (TBB and SPLL-V4), wet or dry season, and early wet, late wet, early dry, and late dry defined as the strata. The median survival time $(\hat{t}(50))$ using this procedure was calculated as the smallest observed time that the estimated survival function was $<0.5$ :

$$
\hat{t}(50)=\min \left\{t_{i} \mid \hat{s}\left(t_{i}\right)<0.5\right\}
$$

Based on the almost 2-to-1 difference in the number of SPLL-V4-infected papaya $(n=167)$ to TBB-infected papaya $(n=85)$, we questioned whether these two phytoplasma strains influenced papaya survival similarly. Examination of the effect of different seasonal periods was based on analysis of contingency tables. We tested the hypothesis that the proportion of newly symptomatic phytoplasma-infected papaya was equal throughout the different seasons, either for two-season (wet/dry) or for fourseason (early wet/late wet/early dry/late dry) systems (FREQ procedure in SAS). In the two-season system, the wet period was defined as the months of October to March, while the dry period was considered the months April to September. The four season strata categorized quarterly periods that newly symptomatic phytoplasma-infected plants were first detected: the early wet period from October to December, the late wet period from January to March, the early dry period from April to June, and the late dry period from July to September.

Modeling time-to-death of papaya plants. We took three approaches to model the relationship between time-to-death and covariates that describe different plant characteristics: Cox proportional hazard models, accelerated failure time analysis, and time-dependent Cox models. The Cox proportional hazard model examines the influence of potential covariates on the hazard of death for an individual $(2,12)$. The hazard at time $t$ is the probability that an individual who has survived to time $t$ will die in the next small period of time $(2,5,12)$. The Cox proportional hazard model assumes the hazards for two groups are proportional (2). The general form for the Cox proportional hazard model is:

$$
h\left(t, x_{i}\right)=e^{f\left(x_{i}\right)} h_{o}(t)
$$

where $e^{f\left(x_{i}\right)}$ indicates how different covariates affect survival (i.e., compares the hazard to the baseline) and $h_{o}(t)$ is an arbitrary baseline hazard function that is assumed to be the same for all groups $(2,12)$. Data were analyzed using the PHREG procedure in SAS. We used likelihood ratio tests to compare the different models to the null model and used a $\chi^{2}$ test to determine if models with additional covariates significantly improve the null model $(2,12)$.

The second modeling approach was accelerated failure time analysis. In accelerated failure time analysis, the main goal is to determine to what extent covariates influence the acceleration of the time-todeath of an individual $(2,5)$. The general accelerated failure time model is:

$$
\log \left(t_{i}\right)=f\left(x_{i}\right)+\varepsilon_{i}
$$

where $f\left(x_{i}\right)$ describes how covariates influence the log time-to-death (i.e., these incorporate our assumptions regarding which covariates are important) and $\varepsilon_{i}$ is the error distribution (5). We first fit a model that incorporated all covariates of interest using the LIFEREG procedure in SAS to examine six distributions in order to determine which distribution best fit the data. The six distributions examined were the Weibull, exponential, log-logistic, lognormal, logis- 
tic, and normal. Distribution selection was based on the log-likelihood values from which we calculated the Akaike Information Criterion (AIC) (20) and selected the distribution that had the smallest value. After the best distribution was selected, the following covariates were added to the model one at a time: (i) phytoplasma strain, (ii) plant age at infection, (iii) season either as wet and dry or early wet, late wet, early dry, late dry. Phytoplasma strain and the two methods to define seasonality were considered classification variables, whereas plant age was considered a continuous variable. Comparisons of the models with covariates to the null model were handled in the same manner as the Cox proportional hazard models.

We also extended the Cox model to incorporate a time-dependent variable to compare the TBB and SPLL-V4 phyto- plasma strains. Using this approach, the assumption of proportional hazards is no longer valid (12). We were interested in determining how the hazard ratio for phytoplasma strain SPLL-V4 versus strain TBB changed over time. We defined the time-dependent variable in two ways. The first approach was to code a phytoplasma strain*time variable, where time was based on when a papaya plant was first observed to be symptomatic and infected using the 3 -month groupings for season described in the Kaplan-Meier section $(n=12$ time points). The second approach was similar to the first, except that we created a phytoplasma strain*time variable using the 6month grouping for season (wet/dry) ( $n=$ 6 time points). We again used the PHREG procedure to model the data. With this approach, the calculation of the estimated hazard ratio is based on:
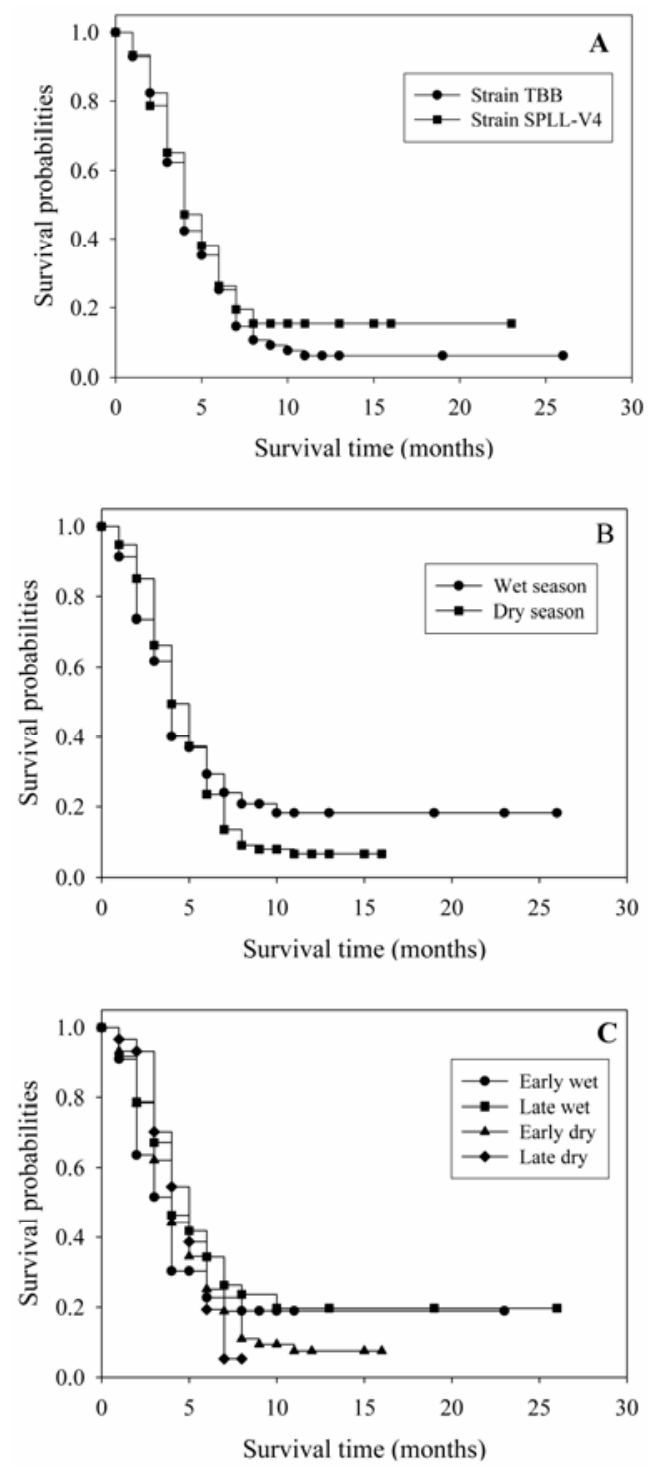

Fig. 1. Plot of survival probabilities using the Kaplan-Meier estimate of the survival function for phytoplasma-infected papaya. A, Comparison of phytoplasma type tomato big bud (TBB) and sweet potato little leaf V4 (SPLL-V4). B, Comparison of papaya plants found infected during the wet season (October to March) and the dry season (April to September). C, Comparison of papaya plants found infected during the early wet season (October to December), late wet season (January to March), early dry season (April to June), and late dry season (July to September).

$$
\mathrm{HR}=\exp \{\hat{\beta}+\lfloor T(t) * \hat{\delta}]\}
$$

where $\mathrm{HR}=$ the estimated hazard ratio, $\hat{\beta}$ $=$ the estimate for phytoplasma strain, $T(t)$ $=$ time-dependent period, and $\hat{\delta}=$ the estimate for strain*time (12). The hazard ratio was calculated as the ratio of SPLL$\mathrm{V} 4 / \mathrm{TBB}$. Values greater than 1 indicated a greater hazard for SPLL-V4 compared with the TBB strain, whereas values less than 1 indicated a greater hazard for the TBB strain. Calculation of the $95 \%$ confidence interval was done using the large sample formula:

$\exp (\{\hat{\beta}+[T(t) \times \hat{\delta}]\} \pm 1.96 \sqrt{\operatorname{Var}\{\hat{\beta}+[T(t) \times \hat{\delta}]}\})$ where the estimated variance was obtained as:

$$
\begin{aligned}
& \operatorname{Var}\{\hat{\beta}+[T(t) \times \hat{\delta}]\}=s_{\hat{\beta}}^{2}+\left\{[T(t)]^{2} \times s_{\hat{\delta}}^{2}\right\} \\
& \quad+[2 \times T(t) \times \operatorname{cov}(\hat{\beta}, \hat{\delta})]
\end{aligned}
$$

where $s_{\hat{\beta}}^{2}$ and $s_{\hat{\delta}}^{2}$ are the estimated variances for strain and strain*time, and $\operatorname{cov}(\hat{\beta}, \hat{\delta})$ is the estimated covariance for the strain and strain*time variables (12).

\section{RESULTS}

Kaplan-Meier estimate of survival function. A total of 85 papaya plants were infected with phytoplasma strain TBB and 167 plants were infected with strain SPLLV4 by April 1999. The first phytoplasmainfected plants were observed from the first assessment (May 1996) and were found throughout the entire study. For TBB-infected papaya, five plants (5.9\%) were censored (still alive) on September 1998, and three plants $(3.5 \%)$ were censored at the end of the study. There were $12.6 \%$ of the SPLL-V4-infected plants censored in September $1998(n=21$ plants), and $15.6 \%$ of the infected plants were censored at the end of the study. Using the Kaplan-Meier approach, the median time-to-death was 4 months for both the TBB and SPLL-V4 strains, and the estimated survival functions were not significantly different (Fig. 1A).

When we examined the seasonal numbers of phytoplasma-infected papaya, a significantly higher number of SPLL-V4 infected papaya plants was observed during the wet season, whereas significantly higher numbers of TBB-infected papaya were observed during the dry season than would be expected to occur by chance $(P \leq$ 0.002 for the wet season versus dry and $P$ $\leq 0.001$ for the early wet/late wet/early dry/late dry seasons) (Tables 1 and 2). Because there were no significant differences in survival times for the TBB and SPLL-V4 phytoplasma strains, and few phytoplasma-infected papaya were observed during some of the seasonal periods, we combined the two phytoplasma strains to also examine the survival curves with season as a stratum. Median survival 
times were both 4 months when comparing the wet and dry season in a two-period approach (Fig. 1B). For four-season periods, median survival times were 4 months for the early wet, late wet, and early dry, compared to 5 months for the late dry seasons (Fig. 1C). There were, however, no significant differences in the estimated survival curves (times) for either the twoseason or the four-season periods.

Modeling survival time using Cox proportional hazards. No significant model improvement was observed $(P>$ $0.20)$ when we compared the different covariates (phytoplasma strain, plant age, two- or four-season periods) to the null model, indicating that these covariates did not affect the hazard for death (survival time) of infected papaya.

Modeling survival time using accelerated failure time. Based on examination of the log-likelihoods and Akaike Information Criterion (AIC) for the full model (all covariates), both the log-logistic and the log-normal models described the survival data better than the other models examined (Table 3). Because there was a questionable convergence of parameter estimates when using SAS for the log-logistic distribution, the log-normal distribution was used to examine the different covariates. The $P$ values for likelihood ratio tests of adding one variable at a time (phytoplasma strain, plant age at time of symptom expression, and the season when a plant was first symptomatic) all exceeded 0.20 ; there was no evidence to support the hypothesis that any of the factors examined reduced survival time for phytoplasma-infected papaya.

Modeling survival time using timedependent Cox models. The estimated hazard ratios for SPLL-V4 over TBB remained constant $(\sim 0.88)$ when using a 3month seasonal period as the timedependent variable (Fig. 2). There appeared to be no evidence, however, that the change in hazard ratio was different from the expected ratio of 1 (i.e., the two hazards were identical). The result for the wet/dry seasonal period analysis was similar (data not shown).

Table 1. Number of infected papaya plants when papaya yellow crinkle symptoms were first observed during the wet (October to March) and dry (April to September) seasons for the tomato big bud (TBB) and sweet potato little leaf V4 (SPLL-V4) phytoplasma strains in the Northern Territory, Australia, during the period May 1996 to April 1999

\begin{tabular}{lcc}
\hline & \multicolumn{2}{c}{ Season } \\
\cline { 2 - 3 } Type & Wet & Dry \\
\hline TBB & $28^{z}$ & 57 \\
SPLL-V4 & 90 & 77 \\
\hline
\end{tabular}

${ }^{\mathrm{z}} \chi^{2}$ test for independence was significantly different from $\propto=0.05\left(\chi^{2}=9.93, P=0.002\right.$, $\mathrm{df}=1)$.

\section{DISCUSSION}

Using survival analysis, we were able to provide a more realistic estimate of postsymptomatic survival time in the papayapapaya yellow crinkle pathosystem. Because Padovan and Gibb (16) did not incorporate censored data to estimate timeto-death, the actual postincubation period survival time of phytoplasma-infected papaya may have been underestimated. Fox (8) indicated that if censored data are not used in analyses, then survival time estimates might be biased. Another advantage in using survival analysis methods is that the effects of other factors on survival times can be investigated. As was previously reported $(15,16)$, time-to-death was calculated only for infected papaya that had died during the study, and thus, postincubation survival times were reported to be less than 4 months for either TBB- or SPLL-V4-infected papaya plants. Because infectious plants (i.e., those with yellow crinkle symptoms that had not yet died) were not incorporated into their analysis, survival times were underestimated by approximately 1 month for both TBB and SPLL-V4 strains. The median survival time estimated by the four survival analysis methods was approximately 2 to 3 weeks longer than the survival times that were calculated using only the papaya plants that had died.

Based on our collective analyses, we can estimate the probable infectious period (incubation period $\left(t_{i}\right)$ plus the postincubation period time-to-death $\left(t_{d}\right)$ ) of phytoplasma-infected papaya by incorporating the estimated mean survival times obtained using the LIFEREG procedure. Across the different strata (variables) examined (phytoplasma strain, wet/dry season, and fourseason periods), the mean survival time ranged from 4.1 to 5.3 months. Although more realistic, these mean survival times probably still underestimated survival times since in all situations, the last observed survival time was only for censored observations. Mean survival time was calculated using the last assessment where death had occurred. However, because the papaya production lifespan is typically 3.5 years, plants that were considered censored at the end of the study (the end of the typical production span) may not be as important if a nonhost crop is planted following papaya. Therefore, by combining incuba- tion periods for TBB and SPLL-V4 (9 to 13 weeks for TBB and 5 to 14 weeks for SPLL-V4) (11) with postincubation survival time, we estimate the overall infectious period for these two phytoplasma strains in papaya ranges from 6 to 9 months.

We have shown that survival time in phytoplasma-infected papaya was not influenced by several risk factors (covariates) that we hypothesized to be associated with plant health (16). The fact that estimated survival times were not significantly different using alternative methods of survival analysis indicates that: (i) phytoplasma strains TBB and SPLL-V4 behave similarly in terms of their effects on papaya survival times after symptom expression, (ii) plant age had no significant influence on survival times, and (iii) seasonal differences concerning when a papaya plant was first symptomatic did not significantly affect papaya survival times. Previous research indicated that the two phytoplasma strains causing yellow crinkle disease (TBB and SPLL-V4) behaved similarly in terms of aggressiveness, defined as the change in disease progress (incidence) with respect to time $(15,16)$. Using survival analysis, we also showed that these two phytoplasma strains behaved similarly in terms of aggressiveness, where aggressiveness was defined in terms of postincubation period survival times for TBB and SPLL-V4.

The results of this study can be used to question the current disease management practices recommended to mitigate yellow crinkle disease of papaya. Current papaya yellow crinkle management programs recommend the ratooning of infected papaya plants (11). However, Guthrie et al. (11) and Elder et al. (6) found that ratooned papaya plants infected with papaya yellow crinkle often exhibited yellow crinkle symptoms in the regrowth. If these plants are still infectious, then there will be little or no effect on delaying the epidemic, except that possibly diseased papaya plants not ratooned represent a larger lesion for acquisition than the developing ratoons. Since papaya plants were infected within the first 6 months of production, papaya yellow crinkle presents a high risk for negative effect on papaya production.

One key assumption with survival analysis is that events (observations) are

Table 2. Number of infected papaya plants when papaya yellow crinkle symptoms were first observed during the early wet (October to December), late wet (January to March), early dry (April to June), and late dry (July to September) seasons for the tomato big bud (TBB) and sweet potato little leaf V4 (SPLL-V4) phytoplasma strains in the Northern Territory, Australia, during the period May 1996 to April 1999

\begin{tabular}{lcccc}
\hline & \multicolumn{4}{c}{ Season } \\
\cline { 2 - 5 } Type & Early wet & Late wet & Early dry & Late dry \\
\hline TBB & $3^{\mathrm{z}}$ & 25 & 35 & 22 \\
SPLL-V4 & 30 & 60 & 40 & 37 \\
\hline
\end{tabular}

${ }^{\mathrm{z}} \chi^{2}$ test for independence was significantly different from $\propto=0.05\left(\chi^{2}=15.62, P=0.001, \mathrm{df}=3\right)$. 
statistically independent. In preliminary that SPLL-V4 was aggregated at distances of 6 to $30 \mathrm{~m}$ from an infected papaya plant, while the spatial pattern for TBB-infected papaya appeared to be random (7). We have also performed exploratory spacetime point process analyses for both SPLL$\mathrm{V} 4$ and $\mathrm{TBB}$, and have found that there is an aggregated spatial and temporal pattern for both SPLL-V4- and TBB-infected papaya (7). However, further work is needed to fully understand the spatiotemporal distribution of infected papaya, although this preliminary spatio-temporal analysis also indicates that ratooning will not be an effective management tactic.

Interestingly, papaya plants were not simultaneously infected by both phytoplasma strains, as the proportion of TBBor SPLL-V4-infected papaya was significantly different when analyzed by seasonal period (Tables 1 and 2). Elder et al. (6) speculated that the three major phytoplasmas of papaya (papaya dieback, papaya yellow crinkle, and papaya mosaic) have different insect vectors, and therefore, this may contribute to the seasonal differences

Table 3. Summary of log-likelihood and Akaike Information Criterion (AIC) used to determine the distribution that best described papaya yellow crinkle survival data and subsequently used to compare accelerated failure time models that incorporated different covariates that potentially influenced survival times of phytoplasmainfected papayas ${ }^{\mathrm{y}}$

\begin{tabular}{lcc}
\hline Distribution & Log-likelihood & AIC $^{\mathbf{z}}$ \\
\hline Exponential & -295.6248 & 593.2496 \\
Weibull & -270.8343 & 545.6686 \\
Log-logistic & -247.6470 & 499.294 \\
Lognormal & -250.8942 & 505.7883 \\
Logistic & -552.0710 & $1,108.142$ \\
Normal & -580.4417 & $1,164.883$ \\
\hline
\end{tabular}

y Distribution selection was based on the smallest AIC value, as well as proper statistical convergence of estimated parameters in the model.

${ }^{\mathrm{z}}$ AIC $=-2(\log$-likelihood $)+2 * k$, where $k=$ penalty for extra parameters. point pattern spatial analyses, it appears

we observed in the proportion of TBB- and SPLL-V4-infected papaya in the Northern Territory. The percentage of SPLL-V4infected papaya (54\%) was much higher during the wet season period (October to March) than the TBB-infected papaya $(33 \%)$. Examination of leafhopper (Orosius spp.) population dynamics (16) indicated a dramatic increase in frequency of Orosius spp. in this plantation between November and December 1996, November and December 1997, and August through December 1998. These periods were immediately before and during the wet season. This time frame also coincides with our defined incubation period for SPLLV4 (5 to 14 weeks) (11). This temporal vector-disease pattern is very similar to observations of Elder et al. (6) in Queensland, Australia, where the majority of papaya plants were infected during September to November. More research, however, is needed to determine the modes of acquisition and transmission of the two phytoplasma strains by different Orosius spp. in order to improve the epidemiological understanding of this pathosystem and to continue to develop the best management tactics (1).

Since it appears that the ecological effect (i.e., time-to-death) of TBB is similar to that of SPLL-V4, it may be of interest to examine host response to these two phytoplasma strains. Because it has been shown that TBB and SPLL-V4 can cause different symptoms (10) and that different insect vectors are probably involved in acquiring and transmitting the two phytoplasma strains (6), it might also be surmised that the host response to the two phytoplasma strains in terms of pathogenesis might also be different. We found this assumption to be false, as the postincubation period survival times were equivalent (equal aggressiveness in the postincubation stage of pathogenesis). Thus, the end result (death) appears to be similar.

Each survival analysis method has its own strengths and weaknesses. The Kaplan-Meier approach is a useful nonpara-

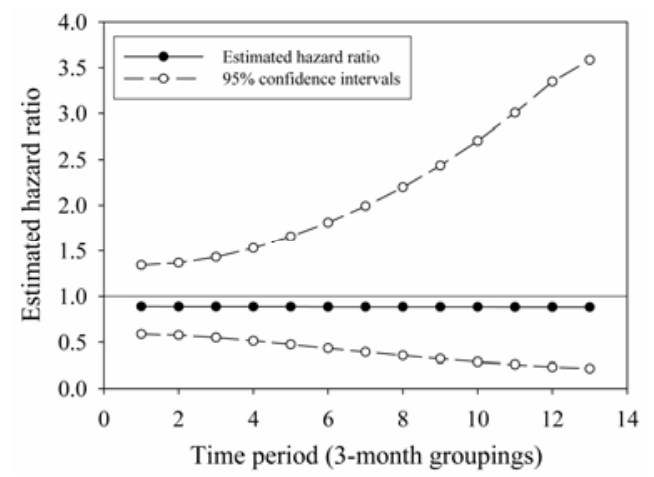

Fig. 2. Plot of the estimated hazard ratio and $95 \%$ confidence interval obtained from a time-dependent Cox model to determine the hazard for the sweet potato little leaf V4 (SPLL-V4) phytoplasma compared with the tomato big bud (TBB) phytoplasma when the time period was 3-month intervals: early wet (October to December), late wet (January to March), early dry (April to June), and late dry (July to September). The line at hazard ratio $=1$ indicates that the hazard for both phytoplasma strains is the same. metric exploratory analysis to examine factors that might be important in influencing the survival probability of an infected plant (2). Cox proportional hazard models are useful when there is no prior information regarding how the biology influences which statistical distribution to select, or if we are interested in whether different management tactics influence the chance of failure $(10,14,18)$. However, if the hazards assumption is violated or untenable, or if we expect management tactics to change the periods of high hazards, then either the time-dependent Cox model or accelerated failure time model may be more appropriate $(8,12,18)$.

Our examination of the full model (all covariates) for accelerated failure time modeling indicated that either the loglogistic or loglinear distributions fit best survival times. Both of these distributional forms are right-skewed distributions and unimodel, and their application to this dataset makes intuitive sense. Collett (2) indicated that if there is an initial increased risk of death, followed by a subsequent decrease in that risk as the patient or plant matures (i.e., the hazard changes directions), then both of these distributional forms will fit better than the Weibull distribution, which is a monotonic function of time. For the papaya plants infected with papaya yellow crinkle, it is likely that if plant death does not occur quickly (within 4 or 5 months), then the risk of death decreases as time increases. With regard to papaya yellow crinkle, the different survival analysis methods have helped us to evaluate the importance of several factors that were hypothesized to potentially influence the time-to-death of postinfection survival of papaya plants, as well as to ascertain the importance of these covariates that might potentially influence the effectiveness of recommended management tactics.

\section{ACKNOWLEDGMENTS}

The first author was supported by a National Science Foundation/Iowa State University Vertical Integration of Graduate Research and Education (VIGRE) Fellowship, under NSF Grant DMS0091953. Research for this project was funded by the Australian Research Council and by funds from the Iowa Agricultural and Home Economics Experiment Station while the fourth author was on Faculty Development Leave in Australia. We thank Mark Gleason for his constructive comments regarding this manuscript.

\section{LITERATURE CITED}

1. Behncken, G. M. 1984. Orosius lotophagorum subsp. ryikyuensis (Hemiptera: Cicadellidae), a new vector of little leaf disease in Australia. Australas. Plant Pathol. 13:35-36.

2. Collett, D. 2003. Modelling Survival Data in Medical Research, 2nd ed. Chapman \& Hall/CRC, Boca Raton, FL.

3. Davis, R. I., Schneider, B., and Gibb, K. S 1997. Detection of differentiation of phytoplasmas in Australia. Aust. J. Agric. Res. 48:535-544.

4. De La Rue, S. J., Schneider, B., and Gibb, K. S. 1999. Genetic variability in phytoplasmas 
associated with papaya yellow crinkle and papaya mosaic diseases in Queensland and the Northern Territory. Australas. Plant Pathol. 28:108-114.

5. Dixon, P. M., and Newman, M. C. 1991. Analyzing toxicity data using statistical models for time-to-death: An introduction. Pages 207-242 in: Metal Ecotoxicology: Concepts and Application. M. C. Newman and A. W. McIntosh, eds. Lewis Publishers, Chelsea, MI

6. Elder, R. J., Milne, J. R., Reid, D. J., Guthrie, J. N., and Persley, D. M. 2002. Temporal incidence of three phytoplasma-associated diseases of Carica papaya and their potential hemipteran vectors in central and south-east Queensland. Australas. Plant Pathol. 31:165-176.

7. Esker, P. D., Gibb, K. S., Dixon, P. M., and Nutter, F. W., Jr. 2005. Use of space-time point process modeling to determine the spatial and temporal dependence of papaya phytoplasmas in Australia. Int. Workshop Plant Dis. Epidemiol. 9th. Landerneau, France.

8. Fox, G. A. 2001. Failure-time analysis. Pages 235266 in: Design and Analysis of Ecological Experiments, 2nd ed. S. M. Scheiner and J. Gurevitch, eds. Oxford University Press, Oxford.
9. Gibb, K. S., Persley, D. M., Schneider, B., and Thomas, J. E. 1996. Phytoplasmas associated with papaya diseases in Australia. Plant Dis. 80:174-178.

10. Gibb, K. S., Schneider, B., and Padovan, A. C. 1998. Differential detection and genetic relatedness of phytoplasmas in papaya. Plant Pathol. 47:325-332.

11. Guthrie, J. N., White, D. T., Walsh, K. B., and Scott, P. T. 1998. Epidemiology of phytoplasma-associated papaya diseases in Queensland, Australia. Plant Dis. 82:1107-1111.

12. Kleinbaum, D. G. 1996. Survival Analysis, A Self-Learning Text. Springer-Verlag, New York

13. Madden, L. V., and Nault, L. R. 1983. Differential pathogenicity of corn stunting mollicutes to leafhopper vectors in Dalbulus and Baldulus species. Phytopathology 73:1608-1614.

14. Muenchow, G. 1986. Ecological use of failure time analysis. Ecology 67:246-250.

15. Nutter, F. W., Jr., Padovan, A. C., and Gibb, K. S. 1999. Epidemiology of two phytoplasma strains associated with yellow crinkle in papaya. Page 57 in: Bienn. Conf. Australas. Plant Pathol. Soc. 12th. Canberra, Australia.
16. Padovan, A. C., and Gibb, K. S. 2001. Epidemiology of phytoplasma diseases in papaya in Northern Australia. J. Phytopathol. 149:649658.

17. Scherm, H. 2002. Epidemiological applications of failure time analysis. (Abstr.) Phytopathology 92:S100-S101.

18. Scherm, H., and Ojiambo, P. S. 2004. Applications of survival analysis in botanical epidemiology. Phytopathology 94:1022-1026.

19. Schneider, B., Seemueller, E., Smart, C. D. and Kirkpatrick, B. C. 1995. Phylogenetic classification of plant pathogenic mycoplasmalike organisms or phytoplasmas. Pages 369381 in: Molecular and Diagnostic Procedures in Mycoplasmology, Vol. 1. S. Razin and J. G. Tully, eds. Academic Press, San Diego.

20. Steel, R. G. D., Torrie, J. H., and Dickey, D. A. 1997. Principles and Procedures of Statistics: A Biometrical Approach, 3rd ed. McGrawHill, Boston, MA

21. Westra, A. A. G., Arneson, C. P., and Slack, S. A. 1994. Effect of interaction of inoculum dose, cultivar, and geographic location on the development of foliar symptoms of bacterial ring rot of potato. Phytopathology 84:410-415. 